\title{
A novel method to limit breast cancer stem cells in states of quiescence, proliferation or differentiation: Use of gel stress in combination with stem cell growth factors
}

\author{
JING WANG ${ }^{1}$, XIANGMING LIU ${ }^{2}$, ZHONGMIN JIANG $^{3}$, LILI LI $^{4}$, \\ ZHIGANG CUI ${ }^{1}$, YUAN GAO ${ }^{1}$, DI KONG ${ }^{1}$ and XIAOZHI LIU ${ }^{5}$ \\ ${ }^{1}$ Department of Tumor Surgery, Tianjin Nankai Hospital, Tianjin 300100; ${ }^{2}$ Department of Esophageal Neoplasms, \\ Cancer Hospital of Tianjin Medical University, Tianjin 300060; ${ }^{3}$ Department of Pathology, Tianjin Fifth Central Hospital, \\ Tianjin 300450; ${ }^{4}$ Department of Orthopedics, Cancer Hospital of Tianjin Medical University, Tianjin 300060; \\ ${ }^{5}$ Central Laboratory, Tianjin Fifth Central Hospital, Tianjin 300450, P.R. China
}

Received November 29, 2015; Accepted May 26, 2016

DOI: $10.3892 / 01.2016 .4757$

\begin{abstract}
The majority of cancer stem cells exist in the G0, or quiescent phase of the cell cycle. However, the cells can escape quiescence following routine radiotherapy and chemotherapy, resulting in tumor recurrence. Presently, achieving the accurate regulation of cancer stem cell growth in order to study a specific state, including the quiescent (mostly G0 or G1 phase), proliferative (mostly S phase) or differential (mostly G2/M phase) states, can be challenging. This makes the determination of cell cycle state-specific characteristics and analysis of potential intervention treatments difficult, particularly for quiescent cells. Breast cancer stem cells were cultured on a soft or hard agar matrix surface in the presence or absence of stem cell growth factors. Cells could be successfully limited in either the quiescent, proliferative or differentiated states. These findings provide a foundation for further study of the cell cycle in breast cancer stem cells.
\end{abstract}

\section{Introduction}

Breast cancer accounts for $22.9 \%$ of invasive cancers in women (1), and $16 \%$ of female cancers in total (2). In 2012, breast cancer accounted for $25.2 \%$ all of cancers diagnosed in women, making it the most common female cancer (3). Over 18 breast cancer sub-types have been described, and these are classified primarily by histological appearance (4). Outcomes for breast cancer vary depending on the cancer type, extent of disease and patient age (5). Current treatment strategies focus on surgery, in combination with radiotherapy and chemotherapy.

Correspondence to: Dr Xiaozhi Liu, Central Laboratory, Tianjin Fifth Central Hospital, 41 Zheijang Road, Tanggu Street, Binhai, Tianjin 300450, P.R. China

E-mail: 1xz7997@126.com

Key words: breast cancer, cell cycle, growth factor, tumor stem cells
However, overall survival and mortality rates, in particular for late-stage breast cancer patients, remain poor (6). A deeper understanding of breast cancer tumorigenesis may aid the development of more effective treatment measures.

The rapid development of gene therapy for numerous cancer types has proceeded in recent years. Much progress has been made towards developing targets within the phosphatidylinositol-3-kinase/protein kinase B (7) and RAS/mitogen-activated protein kinase kinase/extracellular signal-regulated kinase (8) pathways. Additionally, the discovery of the breast cancer stem cell offers an opportunity to potentially cure breast cancer (9-12). The existence of the breast cancer stem cell requires that the mechanisms underlying breast cancer pathogenesis be reconsidered, and doing so may generate novel therapeutic options for breast cancer (13).

Cancer stem cells are a small group of tumor cells with the capacity to proliferate continuously and also differentiate (14). These cells are predominantly in the G0 or resting phase of the cell cycle, and can be activated to proliferate only by external stimulation under optimum conditions (15). The majority of current antitumor drugs target proliferating tumor cells (16). Therefore, these drugs do not kill cancer stem cells (17). Accurately limiting the growth of cancer stem cells in a specific state, including the quiescent, proliferative or differential states, is challenging. This makes the detection of cell characteristics and development of potential intervention treatments difficult, particularly for the quiescent cells.

Breast cancer stem cells were cultured using soft or hard agar as the matrix surface for cell contact in the media. Cells were cultured with or without stem cell growth factors based on the adherence features of the growing cells and the growth maintenance principle of the tumor stem cells (18). Growth indicators of breast cancer stem cells were monitored, including the growth rate of cancer cell clone spheres, the cell cycle, levels of proliferating cell nuclear antigens and telomerase activity. Breast cancer stem cells were successfully limited in either the quiescent, proliferative or differential states, providing valuable references to aid future study of the cell cycle in cancer stem cells. 


\section{Materials and methods}

Cell culture conditions. The breast cancer MDA-MB-231, MDA-MB-435, and MCF-7 cell lines were obtained from American Type Culture Collection (Manassas, VA, USA). Cells were cultured in complete medium consisting of Dulbecco's modified Eagle's medium (Gibco; Thermo Fisher Scientific, Inc., Waltham, MA, USA), containing high glucose and pyruvate and supplemented with $10 \%$ fetal bovine serum (FBS; Invitrogen; Thermo Fisher Scientific, Inc.). Cells were maintained at $37^{\circ} \mathrm{C}$ in a humidified $5 \% \mathrm{CO}_{2}$ atmosphere.

Separation of cluster of differentiation (CD)133-positive breast stem cells. Cells $\left(1 \times 10^{8}\right)$ were suspended in $0.5 \mathrm{ml} \mathrm{PBE}$ incubation solution, consisting of phosphate-buffered saline (PBS), $0.5 \%$ bovine serum albumin (Zhongshan Golden Bridge Biotechnology Co., Ltd., Beijing, China) and 0.08\% ethylenediaminetetraacetic acid ( $\mathrm{pH}$ 7.2), then incubated with a rabbit anti-human CD133 polyclonal antibody $(20 \mu \mathrm{g} / \mathrm{ml}$; dilution, 1:2,000; catalog no. NB120-16518; Novus Biologicals LLC, Littleton, CO, USA) at $4^{\circ} \mathrm{C}$ for $30 \mathrm{~min}$. Antibody-coated superfine magnetic beads (Miltenyi Biotec, Bergisch Gladbach, Germany) were then added, followed by incubation at $10^{\circ} \mathrm{C}$ for $15 \mathrm{~min}$. The cell suspension was then added to the separation column and naturally eluted. The column was rinsed twice with PBS and separated from the magnetic field, inserted into a fresh tube, and 1-2 ml PBE was administered along the needle core to remove the CD133-positive cells. These cells were cultured in neurobasal medium (Invitrogen; Thermo Fisher Scientific, Inc.) containing 1X B27 (Invitrogen; Thermo Fisher Scientific, Inc.), 2 mM L-glutamine, 30 units/ml penicillin-streptomycin (Sigma-Aldrich, St. Louis, MO, USA), $20 \mathrm{ng} / \mathrm{ml}$ basic fibroblast growth factor (bFGF; Miltenyi Biotec), and $20 \mathrm{ng} / \mathrm{ml}$ epidermal growth factor (EGF; Provitro Biosciences, Mt. Vernon, WA, USA).

Preparation of agarose gel matrix medium. Neurobasal medium containing $1 \mathrm{X}$ B27 was prepared, and 0.025 or $3 \mathrm{~g}$ low melting point agarose (Nanjing Sunshine Biotechnology Co., Ltd., Jiangsu, China) was added to $10 \mathrm{ml}$ medium and mixed. The $0.05 \%$ (soft) and 30\% (hard) agarose gel matrix media were stored at room temperature until required. Prior to use, the media were melted and then cooled to $37-39^{\circ} \mathrm{C}$.

Culture of breast cancer stem cell clones. In total, 3 experimental groups were defined: Soft gel+EGF+bFGF; hard gel+EGF+bFGF; and hard gel+2\% fetal bovine serum (FBS) as a control. Agarose gel medium was placed in the bottom of each well in a 48-well plate. Following a 2-h equilibration, the stem cell maintenance factors EGF and bFGF were added to the surface of the gel, and the stem cell maintenance factors were replaced by $2 \%$ FBS onto the surface of the hard gel in the control group. CD133-positive MDA-MB-231, MDA-MB-435 or MCF-7 cells with a sphere diameter of $\sim 25 \mu \mathrm{m}$ were then added to wells. The average sphere diameter was then measured at $0,2,4,6$ and 8 days for analysis of sphere growth.

Telomerase activity. The telomerase activity detection kit (Roche Diagnostics, Indianapolis, IN, USA) was used according to manufacturer's instructions. Briefly, $100 \mu \mathrm{l}$ cell lysate from each sample was incubated for $1 \mathrm{~h}$ at $4^{\circ} \mathrm{C}$ and centrifuged at $12,000 \times \mathrm{g}$ for $15 \mathrm{~min}$. Total messenger RNA (mRNA) was obtained from the upper aqueous phase and $5 \mu \mathrm{l}$ cell extract was used for each polymerase chain reaction (PCR). The PCR conditions were: Primer extension at $25^{\circ} \mathrm{C}$ for $30 \mathrm{~min}$, telomerase inactivation at $94^{\circ} \mathrm{C}$ for $5 \mathrm{~min}$, amplification by 32 cycles of denaturation at $94^{\circ} \mathrm{C}$ for $30 \mathrm{sec}$, annealing at $50^{\circ} \mathrm{C}$ for $30 \mathrm{~s}$, and extension at $72^{\circ} \mathrm{C}$ for $90 \mathrm{sec}$, and a final extension at $72^{\circ} \mathrm{C}$ for $10 \mathrm{~min}$. Finally, $5 \mu \mathrm{l}$ amplification product was mixed with working liquid and substrate following hybridization. The distribution of reaction product bands was analyzed using $1 \%$ agarose gel electrophoresis and the results were visualized under ultraviolet light.

Immunocytochemistry. Immunofluorescence staining of Oct-4 and Ki67 was performed on cell spheres at $72 \mathrm{~h}$ subsequent to inoculation. Cells in 96 -well plates were fixed with $2 \%$ formalin at room temperature, and blocked with $5 \%$ goat serum antigen (Zhongshan Golden Bridge Biotechnology Co., Ltd.). Primary antibodies, mouse anti-human Oct-4 monoclonal antibody (catalog no. sc-5279; Santa Cruz Biotechnology, Inc., Dallas, TX, USA) and mouse anti-human ki-67 monoclonal antibody (catalog no. H00004288-M01; OriGene Technologies, Inc., Beijing, China), were added at a 1:1,000 dilution. Incubation was performed in a moisture box at $4^{\circ} \mathrm{C}$ overnight. Texas-Red (catalog no. sc-474354) or fluorescein isothiocyanate-labeled secondary antibody (dilution, 1:500; Santa Cruz Biotechnology, Inc.) was then added, and 4',6-diamidino-2-phenylindole was used to stain cell nuclei. Image-Pro Plus 6.0 software (Media Cybernetics, Rockville, MD, USA) was used for image analysis.

Flow cytometrycell-cycle analysis. Cell spheres were harvested $72 \mathrm{~h}$ post-inoculation and formed into a single cell suspension. Cells were rinsed and fixed. Following staining with propidium iodide for $30 \mathrm{~min}$, the cell-cycle status was determined by flow cytometry. Flow cytometric analysis was performed by using a FACSCalibur Flow Cytometer (BD Biosciences, Franklin Lakes, NJ, USA). The cell proliferation index $\left(\mathrm{PI}_{\mathrm{x}}\right)$ was calculated using the formula: $\mathrm{PI}_{\mathrm{x}}=\left(\mathrm{S}+\mathrm{G}_{2} \mathrm{M}\right) /\left(\mathrm{G}_{0} \mathrm{G}_{1}+\mathrm{S}+\mathrm{G}_{2} \mathrm{M}\right)$. At least 10,000 PI-negative events were collected for analysis. Acquired data were analyzed using CELLQuest 3.3 software (BD Biosciences).

Western blotting. Cell spheres were cultured in the required media for $72 \mathrm{~h}$. Protein lysates $(15 \mu \mathrm{g})$ were fractionated in 4-20\% sodium dodecyl sulfate polyacrylamide gel electrophoresis gels, transferred to nitrocellulose membranes and blocked with 5\% skimmed milk and $0.1 \%$ Tris-buffered saline with Tween 20 (TBST). Membranes were then incubated with primary antibodies, rabbit anti-human cyclin E1 polyclonal antibody (dilution, 1:2,000; catalog no. sc-198; Santa Cruz Biotechnology, Inc.), rabbit anti-human cyclin D1 polyclonal antibody (dilution, 1:1,000; catalog no. sc-718; Santa Cruz Biotechnology, Inc.) and rabbit anti-human cyclin B1 polyclonal antibody (dilution, 1:2,000; catalog no. sc-752; Santa Cruz Biotechnology, Inc.). Next, membranes were washed five times in $0.1 \%$ TBST and incubated for $1 \mathrm{~h}$, followed by incubation with secondary chicken anti-rabbit immunoglobulin 
A

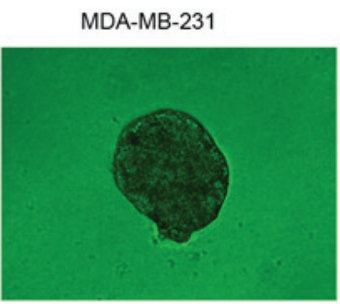

B
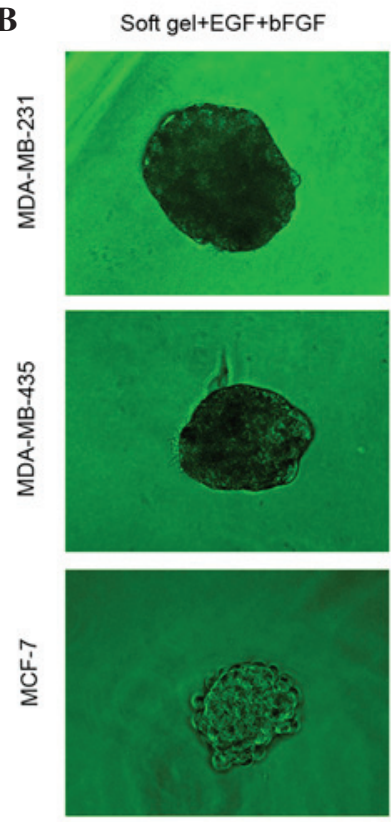

MDA-MB-435

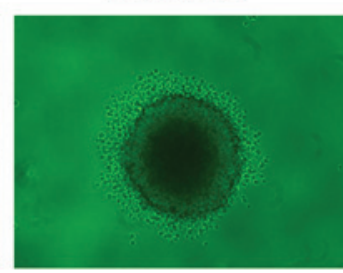

Hard gel+EGF+bFGF
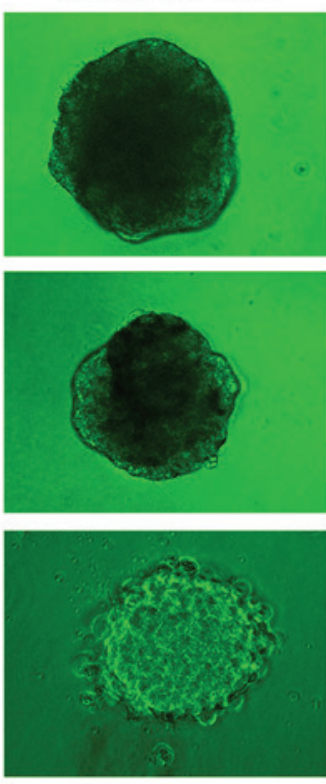

MCF-7
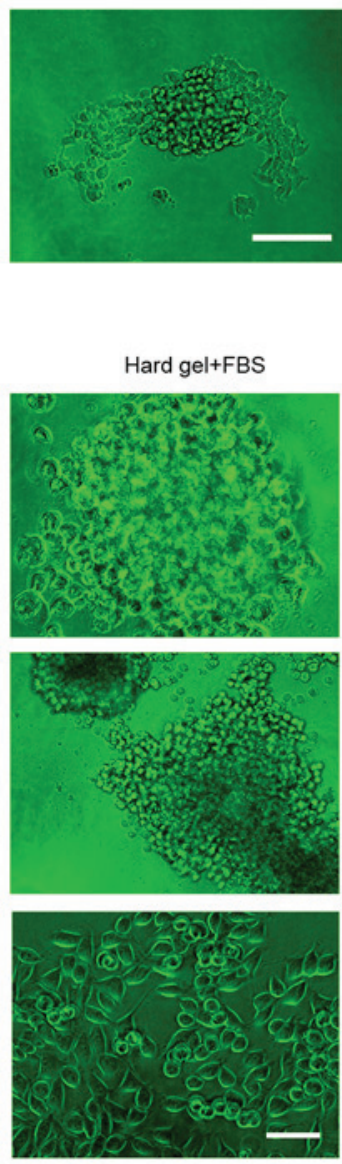

$\mathbf{C}$

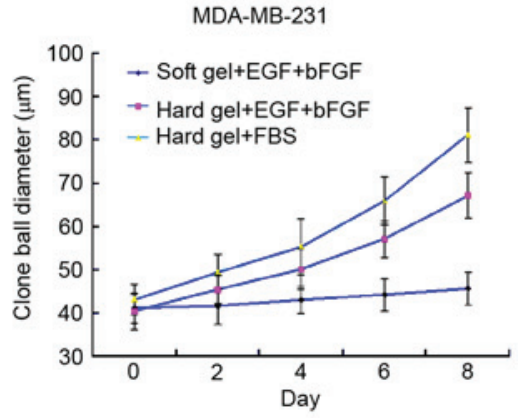

MDA-MB-435

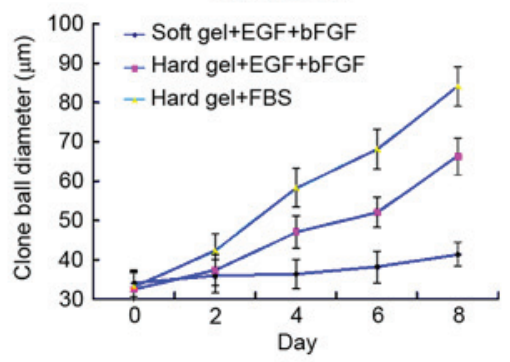

MCF-7

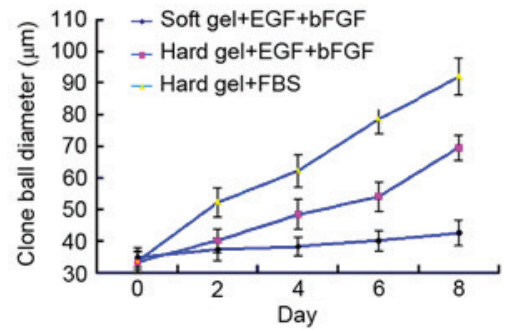

Figure 1. Growth states of breast cancer stem cell clone spheres in a limited culture environment (scale bar, $50 \mu \mathrm{m}$ ). (A) Three CD133-positive breast cancer cell lines were cultured in conventional tumor stem cell culture medium and formed typical and atypical clone balls. Typical and atypical clone balls were transferred to limited culture medium where they showed (B) distinct cellular morphologies and (C) growth velocities. EGF, epidermal growth factor; bFCF, basic fibroblast growth factor; FBS, fetal bovine serum.

(Ig)G horeradish peroxidase (HRP) (dilution, 1:2,000; catalog no. sc-516087; Santa Cruz Biotechnology, Inc.) or chicken anti-goat IgG-HRP (dilution, 1:2,000; catalog no. sc-516086; Santa Cruz Biotechnology, Inc.). The specific protein was detected using a Super Signal protein detection kit (Pierce; Thermo Fisher Scientific, Inc.). The membrane was then stripped and re-probed with a goat anti-human $\beta$-actin polyclonal primary antibody (dilution, 1:1,000; catalo no. sc-1616; Santa Cruz Biotechnology, Inc.).

Statistical analysis. Data are expressed as the mean \pm standard error. Statistical analysis was performed using analysis of variance, $\chi^{2}$ test or Student's $t$-test on the SPSS 11.0 software (SPSS, Inc., Chicago, IL, USA). Statistically significant differences were indicated by $\mathrm{P}<0.05$ or $\mathrm{P}<0.01$.

\section{Results}

Culture of CD133-positive breast cancer stem cell clone spheres. CD133-negative cells were separated by flow cytometry. These cells exhibited no significant change after culture for two weeks and could not form spheres until four weeks (data not shown). CD133-positive breast cancer cells formed evident spheres and gradually grew larger (Fig. 1A). However, not all CD133-positive breast cancer cells grew into typical clone spheres. MDA-MB-231 cells formed round spheres, whereas MDA-MB-435 cells had distinct lace-like extensions surrounding the spheres. MCF-7 cells did not form readily identifiable spheres (Fig. 1A).

Analysis of breast cancer stem cell sphere growth curves in a limited culture environment. The aforementioned clone spheres were transferred to limited culture medium, in which they exhibited distinct cellular morphologies and growth velocities (Fig. 1B and C). When cultured on a soft agar surface supplemented with EGF and bFGF, breast cancer stem cells from all 3 cell lines formed typical spheres, which increased in diameter with incubation time. The diameter of the breast cancer stem cell spheres cultured on hard agar with EGF and bFGF increased relatively more rapidly from 2 days post-inoculation. Additionally, spheres cultured on the hard gel surface with FBS rapidly spread out and expanded, but lost their 3-dimensional structure.

Breast cancer stem cell spheres proliferate in limited culture conditions. The MDS-MB-231 cell line was selected for further follow-up experiments based on the formation of round spheres. Telomerase activity can indirectly indicate 
A

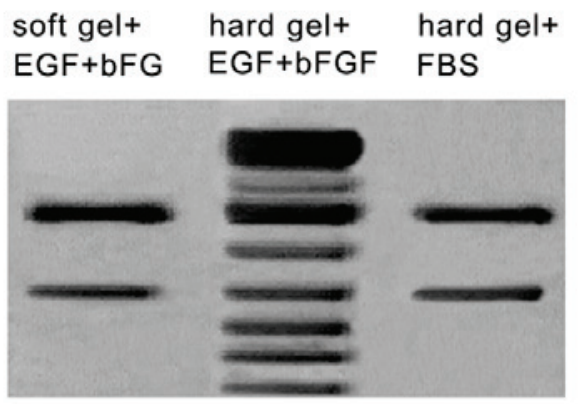

C

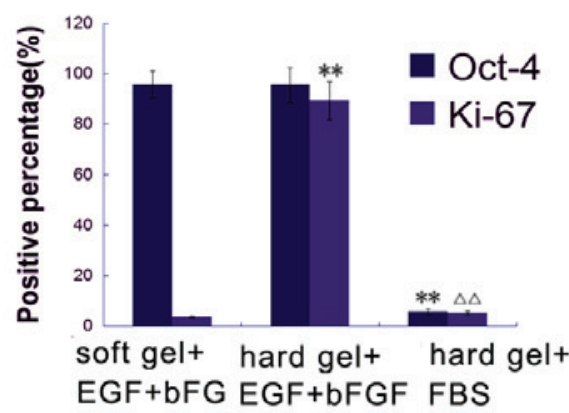

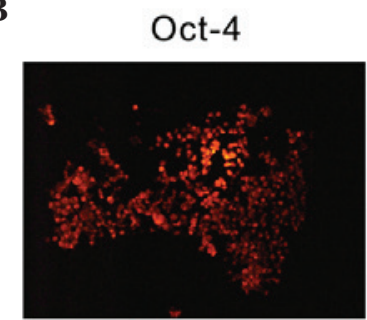
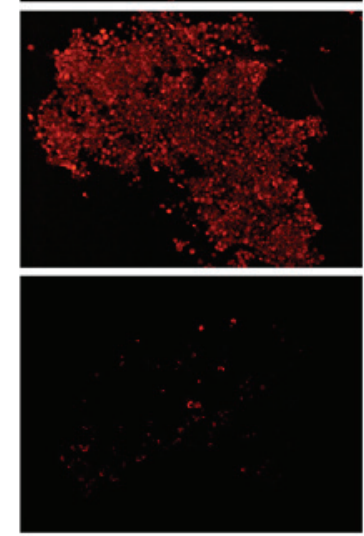

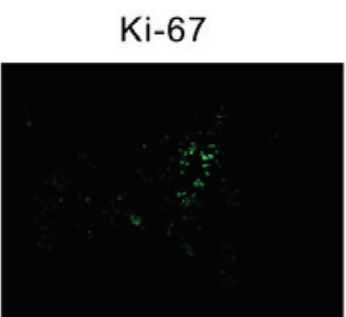

soft gel+

EGF+bFGF

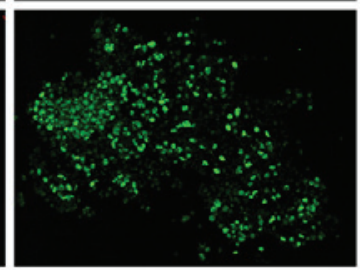

hard gel+

$E G F+b F G F$

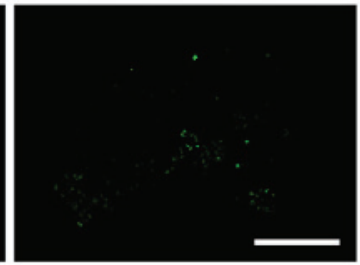

Figure 2. Breast cancer stem cell clone balls exhibited distinct cell proliferation activity in limited culture conditions. (A) Telomerase activities as detected by the repeat amplification of telomerase extension products. (B) Protein expression levels of Oct- 4 and Ki- 67 were detected by immunocytochemistry assay. (C) Values were compared following normalization by cell count. ${ }^{* *} \mathrm{P}<0.01$ vs. soft gel $+\mathrm{EGF}+\mathrm{bFGF} ;{ }^{\Delta \Delta} \mathrm{P}<0.01 \mathrm{vs}$. hard gel $+\mathrm{EGF}+\mathrm{bFGF}$. EGF, epidermal growth factor; bFCF, basic fibroblast growth factor; FBS, fetal bovine serum.

cell proliferation (19). Spheres cultured on a soft agar surface supplemented with EGF and bFGF or a hard agar surface with $2 \%$ FBS possessed minimal telomerase activity (Fig. 2A). However, spheres cultured on hard agar with EGF and bFGF possessed relatively more telomerase activity (Fig. 2A).

When cultured on a soft agar medium with EGF and bFGF, breast cancer stem cell spheres exhibited low proliferative activity. The Ki67-positive expression rate was $3.67 \pm 0.24 \%$, and the majority of cells maintained Oct-4 expression $(95.79 \pm 5.31 \%)$. When cultured on hard agar with EGF and bFGF, breast cancer stem cells exhibited a relatively high proliferative activity, with a Ki67-positive expression rate of $89.39 \pm 7.45 \%$ ( $F=0.013$ vs. soft gel $+\mathrm{EGF}+\mathrm{bFGF}$ ). Only a small proportion of cells were maintained in the stemness state, with an Oct-4-positive rate of $95.71 \pm 6.85 \%$ $(\mathrm{F}=0.455$ vs. soft gel $+\mathrm{EGF}+\mathrm{bFGF})$. When cultured on hard agar plus $2 \%$ FBS, breast cancer stem cells exhibited similarly low proliferative activity, with a Ki67-positive expression rate of $5.29 \pm 0.69 \%(\mathrm{~F}=0.034$ vs. soft gel $+\mathrm{EGF}+\mathrm{bFGF}$; and $\mathrm{F}=0.019$ vs. hard gel $+\mathrm{EGF}+\mathrm{bFGF})$. Oct 4 was expressed in only $5.77 \pm 1.24 \%$ of cells ( $\mathrm{F}=0.014$ vs. soft gel $+\mathrm{EGF}+\mathrm{bFGF}$; and $\mathrm{F}=0.017$ vs. hard gel $+\mathrm{EGF}+\mathrm{bFGF}$ ) (Fig. $2 \mathrm{~B}$ and $\mathrm{C}$ ).

Breast cancer stem cells are held in a specific phase of the cell cycle when cultured in limiting conditions. Flow cytometry revealed that when cultured in soft agar medium with EGF and bFGF, the majority of breast cancer stem cells (75.79\%) were in a non-proliferative phase (G0 or G1; Fig. 3A and B). When cultured in hard agar medium with EGF and bFGF, 48.71\% $(\mathrm{F}=0.031$ vs. soft gel $+\mathrm{EGF}+\mathrm{bFGF})$ of the breast cancer stem cells were in proliferative states (S or G2/M; Fig. 3A and B). While $22.13 \%$ ( $F=0.037$ vs. soft gel $+\mathrm{EGF}+\mathrm{bFGF}$; and $\mathrm{F}=0.029$ vs. hard gel $+\mathrm{EGF}+\mathrm{bFGF})$ of breast cancer stem cells were in the $\mathrm{S}$ phase when cultured on the hard agar surface with $2 \% \mathrm{FBS}$, there were more cells in the $\mathrm{G} 2 / \mathrm{M}$ phase $(18.63 \%$; $\mathrm{F}=0.035$ vs. soft gel $+\mathrm{EGF}+\mathrm{bFGF}$; and $\mathrm{F}=0.027$ vs. hard gel $+\mathrm{EGF}+\mathrm{bFGF}$ ) compared with either of the two other groups.

Western blotting was used to better identify the cell cycle phase distribution. Proteins identified were: Cyclin E1, which accumulates at the G1-S phase boundary and is degraded as cells progress through $\mathrm{S}$ phase (20); cyclin D1, which is synthesized rapidly, accumulates in the nucleus during the G1 phase and is degraded as the cell enters S phase (21); and cyclin B1, which is expressed predominantly during the G2/M phase (22). Cyclin E1 expression was at the lowest level when MDS-MB-231 stem cells were cultured in soft agar medium with EGF and bFGF (Fig. 3C and D). However, the expression peak of cyclin D1 appeared when cells were cultured in hard agar medium with EGF and bFGF. Peak cyclin B1 protein levels were present when cells were cultured on the hard agar surface with 2\% FBS (Fig. 3C and D). These results are consistent with those from the flow cytometry analysis.

\section{Discussion}

Previous studies show that multiple tumor types possess a small population of stem cells that retain the capacity for self-renewal and can differentiate into multiple cell types $(9,11)$. These cells also possess the properties of unlimited proliferation, low differentiation, high invasion and immune evasion $(9,10,13,14)$. Additionally, cancer stem cells can escape death mediated by conventional radiation and chemotherapy treatments, as the majority of cells are in a dormant state (G0). These cells are a source of recurrence following tumor resection surgery $(15,17)$. 
A

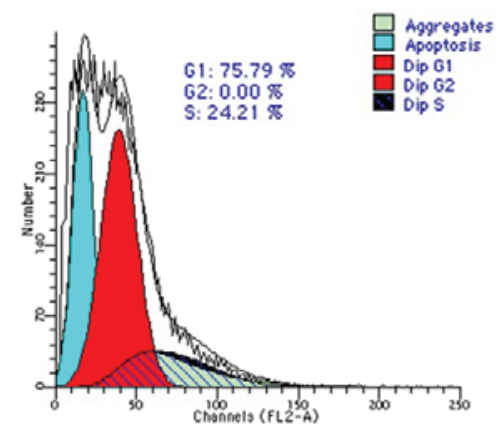

soft gel+EGF+bFGF

B

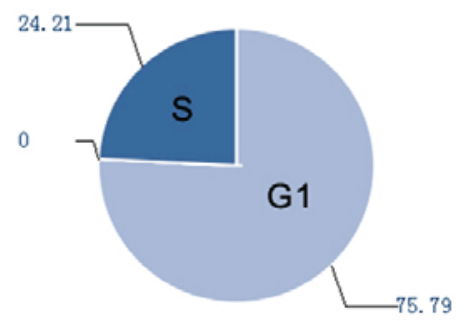

soft gel+EGF+bFGF

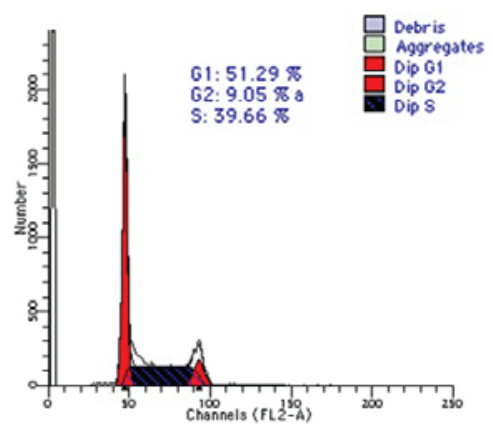

hard gel+EGF+bFGF

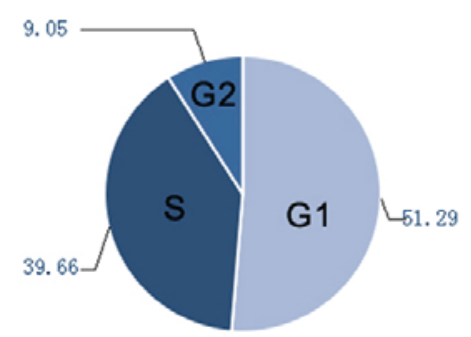

hard gel+EGF+bFGF

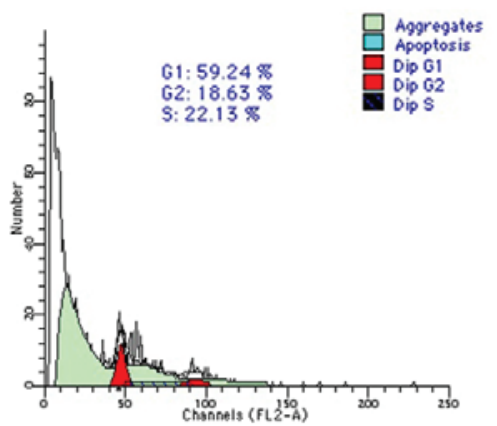

hard gel+FBS

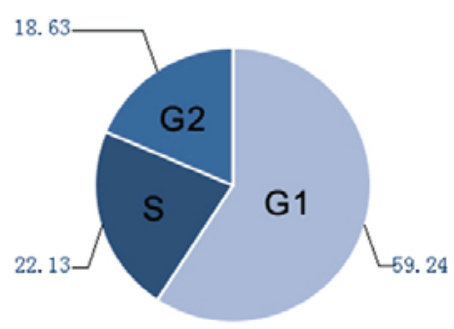

hard gel+FBS

C

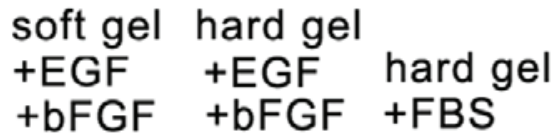

Cyclin E1

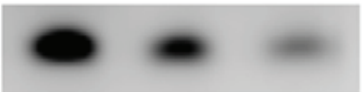

$49 \mathrm{kDa}$

$$
\beta \text {-actin }
$$

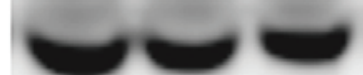

Cyclin B1

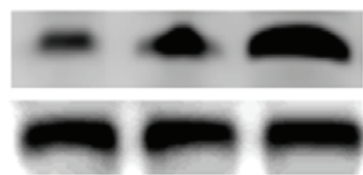

$\beta$-actin

Cyclin D1

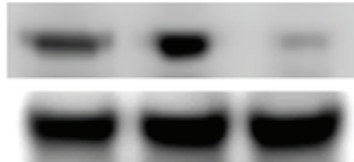

$49 \mathrm{kDa}$

$43 \mathrm{kDa}$

$60 \mathrm{kDa}$

$43 \mathrm{kDa}$

$33 \mathrm{kDa}$

$43 \mathrm{kDa}$ $\beta$-actin

$43 \mathrm{kDa}$
D soft gel+EGF+bFGF

$\square$ hard gel+EGF+bFGF

$\square$ hard gel+ FBS

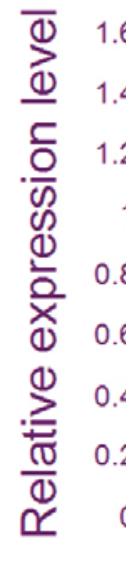

Figure 3. Breast cancer stem cells were restricted to a specific phase of cell cycle when cultured in limiting conditions. (A) Results of the cell cycle analysis, detected by flow cytometry. (B) Pie chart of cell cycle analysis data in part A. (C) Expression of cell-cycle protein markers by western blotting. (D) Graphical presentation of protein expression data in part $\mathrm{C}$. ${ }^{*} \mathrm{P}<0.05$ and ${ }^{* *} \mathrm{P}<0.01$ vs. soft gel $+\mathrm{EGF}+\mathrm{bFGF} ;{ }^{\Delta} \mathrm{P}<0.05$ and ${ }^{\Delta \Delta} \mathrm{P}<0.01$ vs. hard gel $+\mathrm{EGF}+\mathrm{bFGF}$. EGF, epidermal growth factor; bFCF, basic fibroblast growth factor; FBS, fetal bovine serum.

While numerous studies of cancer stem cells exist, few reports have examined the limitation of cancer stem cells to specific phases of the cell cycle, including the quiescent, proliferative and differential states. Typically, bFGF and EGF are added to the culture medium to maintain the 'stemness' properties of cancer stem cells (23). However, cultured stem cells are commonly in a state of rapid proliferation, and cells in a quiescent state are challenging to observe and detect (24). Park et al have shown that the hardness of the cell adhesion material can affect stem cell proliferation and differentiation (25). Additionally, stem cells exhibit slow expansion speed, fewer stress fibers and a lower growth rate when cultured on the surface of a soft matrix (26). Therefore, the present study attempted to maintain stem cell growth under adherent conditions.

Breast cancer stem cells were cultured on soft and hard agar contact surfaces using culture medium with or without stem cell growth factors added. CD133-positive cancer stem cell spheres were obtained from the MDA-MB-231, MDA-MB-435 and MCF-7 breast cancer cell lines. Inconsistent with the classical 
theory (9), not all CD133-positive breast cancer cells could form typical clone spheres. Only the MDA-MB-231 stem cells were found to form typical round spheres, while MCF-7 stem cells did not adopt a spherical structure. Therefore, improvements to medium supplementation and novel methods for the culture of cancer stem cells are required.

Breast cancer stem cells grew constantly with incubation time only when cultured on the hard agar surface with the addition of bFGF and EGF. Breast cancer stem cell spheres grew slowly on the soft agar surface, even in the presence of EGF and bFGF. While spheres cultured on the hard agar surface with $2 \%$ FBS were slightly enlarged, it is possible that the enlarged tumor cell spheres were as a result of a single differentiated cell of increased size, rather than an increased number of individual tumor stem cells. This would be consistent with a previous report (27).

Breast cancer stem cells cultured on soft agar plus EGF and bFGF exhibited almost no telomerase activity. These cells were in the G1 phase and had low Ki67 expression and increased expression of the typical tumor stem cell marker protein, Oct-4 (28). As flow cytometry cannot distinguish G0 from G1, and G0 may be regarded as an extended G1 state (29), these breast cancer stem cells can be assumed to be in a quiescent state. Contrastingly, breast cancer stem cells cultured on hard agar plus EGF and bFGF exhibited high telomerase activity. These cells were in a proliferative state, expressing high levels of Ki67, Oct-4 and cyclin D1. Breast cancer stem cells cultured on a hard agar surface with $2 \%$ FBS had low telomerase activity, and few cells were in a proliferative state. These cells had decreased expression levels of Ki67 and Oct-4, but increased expression levels of cyclin B1, indicating a differentiated state.

Breast cancer stem cells may be held in a state of quiescence, proliferation or differentiation depending upon adherent growth and the maintenance of the stem cell growth factors. The findings of the present study enhance the basic knowledge of cancer stem cells, particularly those in a quiescent state.

\section{Acknowledgements}

The present study was supported by the China National Natural Scientific Fund, Beijing, China (grant no., 81471175) and the Tianjin Health Bureau Science and Technology Projects, Tianjin, China (grant no., 2014KY23).

\section{References}

1. Rothschild E and Banerjee D: Subverting subversion: A review on the breast cancer microenvironment and therapeutic opportunities. Breast Cancer (Auckl) 9 (Suppl 2): S7-S15, 2015.

2. Wen KY, Fang CY and Ma GX: Breast cancer experience and survivorship among Asian Americans: A systematic review. J Cancer Surviv 8: 94-107, 2014.

3. Gupta A, Shridhar K and Dhillon PK: A review of breast cancer awareness among women in India: Cancer literate or awareness deficit? Eur J Cancer 51: 2058-2066, 2015.

4. Alizadeh F, Bolhassani A, Khavari A, Bathaie SZ, Naji T and Bidgoli SA: Retinoids and their biological effects against cancer. Int Immunopharmacol 18: 43-49, 2014.

5. Gerber B, Marx M, Untch M and Faridi A: Breast reconstruction following cancer treatment. Dtsch Arztebl Int 112: 593-600, 2015.
6. Danilak M and Chambers CR: Adherence to adjuvant endocrine therapy in women with breast cancer. J Oncol Pharm Pract 19: 105-110, 2013.

7. Krastev TK, Jonasse Y and Kon M: Oncological safety of autologous lipoaspirate grafting in breast cancer patients: A systematic review. Ann Surg Oncol 20: 111-119, 2013.

8. Alkatout I, Order B, Klapper W, Weigel MT, Jonat W, Schaefer FK, Mundhenke C and Wenners A: Surgical impact of new treatments in breast cancer. Minerva Ginecol 65: 363-383, 2013.

9. Hong IS, Jang GB, Lee HY and Nam JS: Targeting cancer stem cells by using the nanoparticles. Int J Nanomedicine 10: 251-260, 2015.

10. Bao B, Azmi AS, Ali S, Zaiem F and Sarkar FH: Metformin may function as anti-cancer agent via targeting cancer stem cells: The potential biological significance of tumor-associated miRNAs in breast and pancreatic cancers. Ann Transl Med 2: 59, 2014.

11. Patel S, Shah K, Mirza S, Daga A and Rawal R: Epigenetic regulators governing cancer stem cells and epithelial-mesenchymal transition in oral squamous cell carcinoma. Curr Stem Cell Res Ther 10: 140-152, 2015.

12. Menendez JA and Alarcón T: Metabostemness: A new cancer hallmark. Front Oncol 4: 262, 2014.

13. Anfuso B, Tiribelli $\mathrm{C}$ and Sukowati $\mathrm{CH}$ : Recent insights into hepatic cancer stem cells. Hepatol Int 8 (Suppl 2): S458-S463, 2014.

14. Cherciu I, Bărbălan A, Pirici D, Mărgăritescu C and Săftoiu A: Stem cells, colorectal cancer and cancer stem cell markers correlations. Curr Health Sci J 40: 153-161, 2014.

15. Adorno-Cruz V, Kibria G, Liu X, Doherty M, Junk DJ, Guan D, Hubert C, Venere M, Mulkearns-Hubert E, Sinyuk M, et al: Cancer stem cells: Targeting the roots of cancer, seeds of metastasis and sources of therapy resistance. Cancer Res 75: 924-929, 2015.

16. Stecklein SR, Jensen RA and Pal A: Genetic and epigenetic signatures of breast cancer subtypes. Front Biosci (Elite Ed) 4: 934-949, 2012.

17. Mannelli G and Gallo O: Cancer stem cells hypothesis and stem cells in head and neck cancers. Cancer Treat Rev 38: 515-539, 2012.

18. Podberezin M, Wen J and Chang CC: Cancer stem cells: A review of potential clinical applications. Arch Pathol Lab Med 137: 1111-1116, 2013.

19. Zvereva MI, Shcherbakova DM and Dontsova OA: Telomerase: Structure, functions, and activity regulation. Biochemistry (Mosc) 75: 1563-1583, 2010.

20. Blomme J, Inzé D and Gonzalez N: The cell-cycle interactome: A source of growth regulators? J Exp Bot 65: 2715-2730, 2014.

21. Sheppard KE and McArthur GA: The cell-cycle regulator CDK4: An emerging therapeutic target in melanoma. Clin Cancer Res 19: 5320-5328, 2013.

22. Poss ZC, Ebmeier CC and Taatjes DJ: The Mediator complex and transcription regulation. Crit Rev Biochem Mol Biol 48: 575-608, 2013.

23. Safa AR, Saadatzadeh MR, Cohen-Gadol AA, Pollok KE and Bijangi-Vishehsaraei K: Glioblastoma stem cells (GSCs) epigenetic plasticity and interconversion between differentiated non-GSCs and GSCs. Genes Dis 2: 152-163, 2015.

24. Suhardja A and Hoffman H: Role of growth factors and their receptors in proliferation of microvascular endothelial cells. Microsc Res Tech 60: 70-75, 2013.

25. Park J, Kim HN, Kim DH, Levchenko A and Suh KY: Quantitative analysis of the combined effect of substrate rigidity and topographic guidance on cell morphology. IEEE Trans Nanobioscience 11: 28-36, 2012.

26. Qin L, Huang J, Xiong C, Zhang Y and Fang J: Dynamical stress characterization and energy evaluation of single cardiac myocyteactuating on flexible substrate. Biochem Biophys Res Commun 360: 352-356, 2007.

27. Kumar P, Kadakol A, Shasthrula PK, Mundhe NA, Jamdade VS, Barua CC and Gaikwad AB: Curcumin as an adjuvant to breast cancer treatment. Anticancer Agents Med Chem 15: 647-656, 2015.

28. Zeineddine D, Hammoud AA, Mortada M and Boeuf $\mathrm{H}$ : The Oct4 protein: More than a magic stemness marker. Am J Stem Cells 3: 74-82, 2014.

29. Lyons AB, Blake SJ and Doherty KV: Flow cytometric analysis of cell division by dilution of CFSE and related dyes. Curr Protoc Cytom 9: Unit9.11, 2013. 